06

\title{
Численное исследование ударно-волнового нагружения металлических композитов на базе W и WC
}

\author{
(C) К.К. Маевский ${ }^{1,2}$ \\ ${ }^{1}$ Институт гидродинамики им. М.А. Лаврентьева СО РАН, \\ 630090 Новосибирск, Россия \\ ${ }^{2}$ Новосибирский государственный университет, \\ 630090 Новосибирск, Россия \\ e-mail: konstantinm@hydro.nsc.ru
}

Поступило в Редакцию 16 октября 2020 г.

В окончательной редакции 30 ноября 2020 г.

Принято к публикации 7 декабря 2020 г.

\begin{abstract}
Представлены результаты численных экспериментов по моделированию ударно-волнового нагружения семейства металлических композитов - элконитов, которые представляют собой спеченные тугоплавкие материалы (вольфрам, карбид вольфрама или молибден) в сочетании с легкоплавким металлом - серебром или медью. Использованы термодинамически-равновесная модель ТЕС (thermodynamic equilibrium components). Получено хорошее согласие модельных расчетов с данными разных авторов, которые определены на основе эксперимента. Модель описывает динамические нагружения сплошных и пористых сплавов различных составов таких, как $\mathrm{Cu}-\mathrm{W}, \mathrm{Ag}-\mathrm{W}, \mathrm{Cu}-\mathrm{WC}, \mathrm{Ag}-\mathrm{WC}$, и позволяет осуществлять целенаправленный выбор составов материалов различных значений пористости и соотношений их компонентов с целью получения заданных характеристик при ударно-волновом нагружении сплошных и пористых образцов.
\end{abstract}

Ключевые слова: ударная адиабата, сплавы, вольфрам, термодинамическое равенство, уравнение состояния.

DOI: 10.21883/JTF.2021.05.50694.293-20

\section{Введение}

При развитии метода ударно-волнового синтеза необходимо целенаправленно изменять термодинамические параметры для реализации необходимых условий создания материалов с заданными свойствами. Для этого проводятся исследования сжимаемости многокомпонентных материалов различных составов, при этом большой интерес вызывают группы композитов, элементы которых состоят в основном из тугоплавких металлов вольфрама, молибдена и карбида вольфрама в сочетании с медью [1-11]. Интерес к исследованиям сжимаемости таких образцов связан как с возможностью создания материалов с необходимыми свойствами, так и со свойствами компонентов, входящих в их состав. Комбинации этих элементов создают плотные твердые металлы с превосходной износостойкостью и прочностью при повышенных температурах в сочетании с хорошей тепловой и электрической проводимостью.

При моделировании ударно-волнового нагружения многокомпонентных материалов одним из распространенных подходов является смесевой метод, при котором используется правило аддитивности удельных объемов [12,13]. В этом случае объем ударно сжатого материала предполагается равным сумме объемов компонентов, полученных при том же давлении при их раздельном сжатии. Такой подход упрощает расчеты, однако позволяет достоверно описывать только высокоплотные смеси в ограниченном диапазоне давлений. Как отмечается в $[6,12]$, аддитивное приближение хорошо работает для ряда сплавов, однако в некоторых случаях имеются отклонения расчетных результатов и данных, полученных на основании экспериментов. Причина в том, что такой подход предполагает тождественность процессов сжатия компонентов, находящихся в гетерогенных смесях, и ударного сжатия гомогенных компонентов [6]. В частности, для элконитов $\mathrm{Cu}-\mathrm{W}$ [2] при моделировании в ряде случаев было получено систематическое отклонение расчетных данных от данных, полученных на основании экспериментов [6]. Такие отклонения появляются для образцов при значении пористости $m$ более 1.02 , где $m$ определяется как отношение плотности монолитного образца к плотности исследуемого материала. При этом, как отмечается в [2], экспериментальные данные и для сплошного элконита $\mathrm{Cu}-\mathrm{WC}$ хуже согласуются с расчетными данными, которые выполнены по смесевому методу.

Для учета взаимодействия компонентов смесей была предпринята попытка ввести дополнительно турбулентную энтропию, используя ТЕ-метод [14]. Однако и этот подход не позволяет описывать данные экспериментов работы [13] во всем диапазоне приведенных значений давления. Принципиально новая модель многокомпонентной среды, содержащая новую универсальную форму обмена импульсом и энергией между компонентами (так называемая кластерная форма), предложена и обоснована В.Ф. Куропатенко [15]. В новой модели законы сохранения смеси получаются путем суммирования законов сохранения компонентов. В этом случае для данных [13], как отмечается в [16], „теоретические 
зависимости давления от волновой скорости без учета кластерного взаимодействия лежат выше экспериментальных. Теоретические ударные адиабаты с учетом кластерного взаимодействия лежат ниже экспериментальных адиабат“.

Таким образом, несмотря на большое внимание и практический интерес, остается актуальной задача построения модели, достоверно описывающей термодинамические параметры композитных материалов различных значений пористости.

\section{1. Модель расчета}

Для описания термодинамических параметров сплавов и смесей при ударно-волновом воздействии используется модель ТЕС ударно-волнового нагружения с учетом наличия газа в порах $[17,18]$. Данная модель позволяет описать термодинамические параметры в широком диапазоне давлений при ударно-волновом нагружении для различных составов смесей и соотношений их компонентов [19-21], в том числе и тех смесей, компоненты которых испытывают полиморфный фазовый переход. Модель основана на предположении, что при ударноволновом нагружении все компоненты исследуемого образца находятся в термодинамическом равновесии. Предполагается равенство скоростей, давлений и температур. Для расчета ударно-волнового воздействия на такие образцы используются параметры уравнения состояния только их компонентов. При моделировании поведения конденсированных фаз используются уравнения состояния типа Ми-Грюнайзена. Выписываются термические и калорические формы уравнения состояния

$$
\begin{gathered}
P(\rho, T)=P_{C}(\rho)+P_{T}(T), \quad E(\rho, T)=E_{C}(\rho)+E_{T}(T), \\
P_{T}(\rho, T)=\Gamma \rho E_{T}(T), \quad E_{T}(T)=c_{V}\left(T-T_{0}\right) .
\end{gathered}
$$

Здесь $P_{C}, E_{C}, P_{T}, E_{T}$ - потенциальные и тепловые составляющие давления и удельной энергии, $c_{V}-$ удельная теплоемкость, $T_{0}-$ начальная температура. Для описания поведения многокомпонентных материалов различной пористости в уравнении состояния вводится функция $\Gamma=P_{T} V / E_{T}$, определяющая вклад тепловых компонент, зависящая при этом только от температуры в следующем виде [18]:

$$
\begin{gathered}
\Gamma(T)=\left[\left(\Gamma\left(T_{0}\right)-\Gamma\left(T_{\infty}\right)\right)^{-1}+C\left(T-T_{0}\right)\right]^{-1}+\Gamma\left(T_{\infty}\right), \\
C=\left(\left[\Gamma\left(T_{*}\right)-\Gamma_{\infty}\right]^{-1}-\left[\Gamma\left(T_{0}\right)-\Gamma_{\infty}\right]^{-1}\right)\left(T_{*}-T_{0}\right)^{-1} .
\end{gathered}
$$

Начальная энергия вещества $E_{0}$ при нормальных условиях полагается равной нулю. Термические и калорические формы уравнения состояния конденсированного компонента имеют следующий вид:

$$
\begin{gathered}
P(\rho, T)=A\left(\left(\rho / \rho_{0}\right)^{n}-1\right)+\Gamma c_{V}\left(T-T_{0}\right) \rho \\
E(\rho, T)=A / \rho_{0}\left(\left(\rho / \rho_{0}\right)^{n-1} /(n-1)+\rho_{0} / \rho-n /(n-1)\right) \\
+c_{V}\left(T-T_{0}\right) .
\end{gathered}
$$

Здесь $\rho, \rho_{0}-$ текущая и начальная плотности. Для газа берется уравнения состояния идеального газа

$$
P=(\gamma-1) \rho_{g} c_{g}\left(T-T_{0}\right),
$$

где $\rho_{g}$ - плотность газа, а постоянные $\gamma=1.41-$ показатель адиабаты и $c_{g}=718 \mathrm{~J} /(\mathrm{kg} \cdot \mathrm{K})$ - теплоемкость газа - соответствуют воздуху аналогично [22]. На фронте волны выписываются условия сохранения потока массы для каждого компонента смеси и сохранения потоков импульса и энергии для смеси в целом. При таком подходе для образца, включающего в свой состав $n$ конденсированных компонент, можно получить следующее выражение в предположении, что $P_{0}=0$ :

$$
P=\frac{\sum_{i=1}^{n} A_{i} \frac{\mu_{i 0}}{\sigma_{i}}\left[\left(h_{i}-\frac{k_{i}+1}{k_{i}-1}\right) \sigma_{i}^{k_{i}}+\frac{2 k_{i} \sigma_{i}}{k_{i}-1}-h_{i}-1\right]}{\sum_{i=1}^{n} \frac{\mu_{i 0}}{\sigma_{i}} h_{i}+\frac{h_{g}}{\sigma_{g}}\left(1-\sum_{i=1}^{n} \mu_{i 0}\right)-1} .
$$

Здесь $h_{i}=2 / \Gamma_{i}+1, i=1, \ldots, n ; h_{g}=2 /(\gamma-1)+1$. При этом $\mu_{10}$ - объемная доля соответствующего компонента, $\rho_{i} / \rho_{i 0}-$ его текущая и начальная плотности, $\sigma_{i}=\rho_{i} / \rho_{i 0}$ - степени его сжатия, а $\rho_{g}, \rho_{g 0}$, $\sigma_{g}=\rho_{g} / \rho_{g 0}-$ соответствующие плотности и сжатие газа. Добавляя к (1) соотношения, которые выражают равенство температур всех компонентов, в совокупности с уравнениями состояния каждого компонента можно построить зависимости $P(U)$ и $D(U)(U, D-$ давление, массовая и волновая скорости), рассматривая их как ударные адиабаты многокомпонентного материала $[17,18]$. Для сплошного материала $\sum_{i=1}^{n} \mu_{i 0}=1$, что соответствует объемной доли газа, равной нулю. Чистое вещество можно рассматривать как образец с одним конденсированным компонентом.

\section{2. Результаты моделирования}

Данные, полученные на основании экспериментов для различных по составам и значениям пористости $m$ из $[23,24]$ композитов, состоящих из $\mathrm{W}$ и $\mathrm{Cu}$, и результаты моделирования термодинамических параметров для данных материалов приведены на рис. 1 в переменных давление $P$ - сжатие $\sigma$; на рис. $2-$ в переменных волновая $D$ и массовая $U$ скорости.

Учет пористости в рамках рассматриваемой модели для смеси меди и вольфрама позволил достоверно описать имеющиеся экспериментальные данные без сушественных погрешностей для всех значений пористости. На рис. 1,2 представлены ударные адиабаты образцов следующих составов с указанием весовых долей компонентов и значений пористости $m$ : Elkonite $2125 \mathrm{C}, \mathrm{W}(25) \mathrm{Cu}(75) m=1.014$; Elkonite $1 \mathrm{~W} 3$, $\mathrm{W}(55) \mathrm{Cu}(45), m=1.0195$; Elkonite $3 \mathrm{~W} 3, \mathrm{~W}(68) \mathrm{Cu}(32)$, $m=1.022$, Elkonite $10 \mathrm{~W} 3, \mathrm{~W}(76) \mathrm{Cu}(24), m=1.068$. Для наглядности расчетные кривые и данные, полученные 
Значения определяющих параметров

\begin{tabular}{c|c|c|c|c|c|c|c|c}
\hline Материал & $A, \mathrm{GPa}$ & $\rho, \mathrm{g} / \mathrm{cm}^{3}$ & $n$ & $c_{v}, \mathrm{~J} /(\mathrm{kg} \cdot \mathrm{K})$ & $\Gamma\left(T_{0}\right)$ & $\Gamma\left(T_{*}\right)$ & $T_{*}, \mathrm{~K} \cdot 10^{3}$ & $\Gamma\left(T_{\infty}\right)$ \\
\hline $\mathrm{Ag}$ & 36.71 & 10.460 & 3.5 & 277 & 2.47 & 1.65 & 23 & 0.500 \\
\hline $\mathrm{Cu}$ & 34.83 & 8.930 & 4.00 & 380 & 2.00 & 1.50 & 23 & 0.510 \\
\hline $\mathrm{W}$ & 101.8 & 19.235 & 3.105 & 140 & 1.61 & 1.35 & 23 & 0.400 \\
\hline $\mathrm{WC}$ & 200 & 15.660 & 2.40 & 217 & 1.80 & 1.60 & 23 & 0.500
\end{tabular}

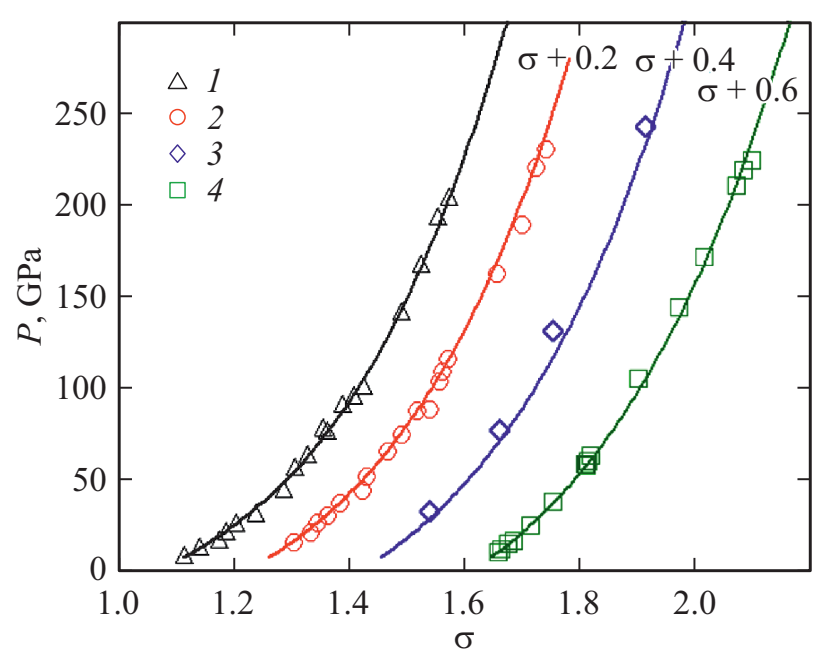

Рис. 1. Ударные адиабаты и данные [23] для пористой смеси W и $\mathrm{Cu}, m: 1-1.014,2-1.0195,3-1.022,4-1.068$.

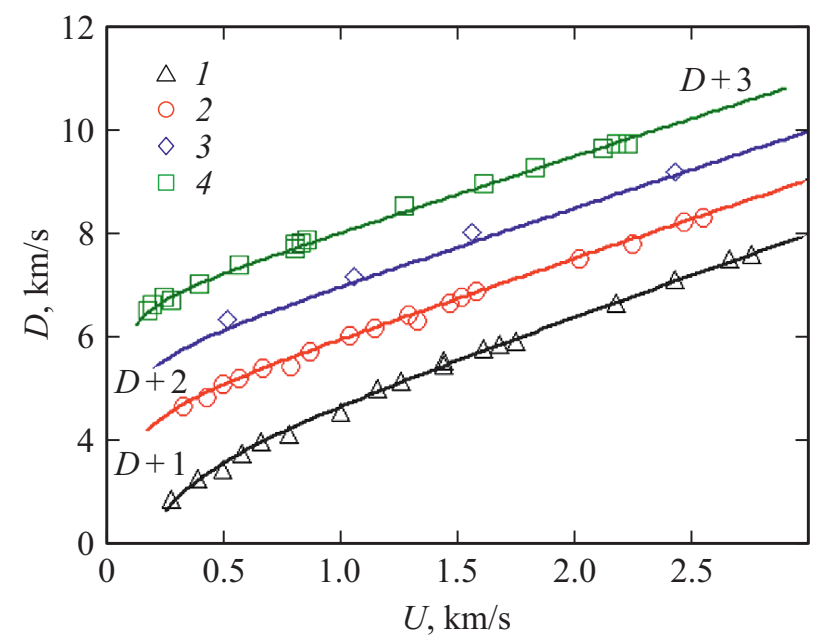

Рис. 2. Расчетные (линии) и экспериментальные (точки) зависимости волновой скорости от массовой скорости. Обозначения те же, что на рис. 1 .

на основании экспериментов, приведены со сдвигом 0.2 по значению сжатия на рис. 1 и со сдвигом $1 \mathrm{~km} / \mathrm{s}$ по значениям волновой скорости на рис. 2. Показано хорошее соответствие модельных расчетов с данными разных авторов, которые определяются на основе эксперимента, при этом отсутствует систематическое отклонение для расчетных данных с данными экспериментов, которое возникало при использовании других моделей $[3,6]$. Параметры, полученные на основе экспериментальных данных [23-27] для компонентов, входящих в состав исследуемых материалов, приведены в таблице.

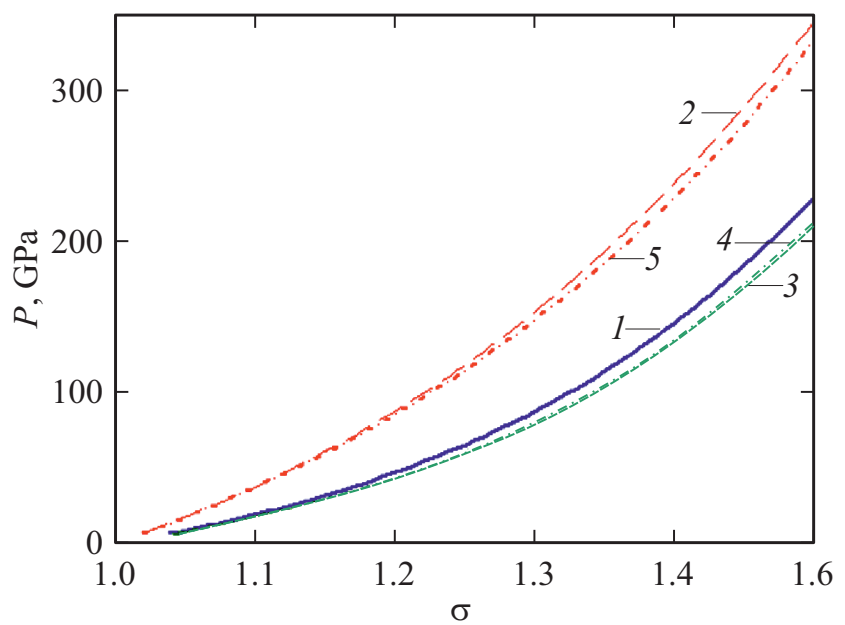

Рис. 3. Сжатие материала и компонентов вдоль ударной адиабаты. Расчет: 1 - сжатие смеси Elkonite 2125С в целом, 2 - сжатие чистого $\mathrm{W}(m=1.068), 3$ - сжатие чистой $\mathrm{Cu}$ $(m=1.068), 4-$ сжатие $\mathrm{Cu}$ в смеси, $5-$ сжатие $\mathrm{W}$ в смеси.

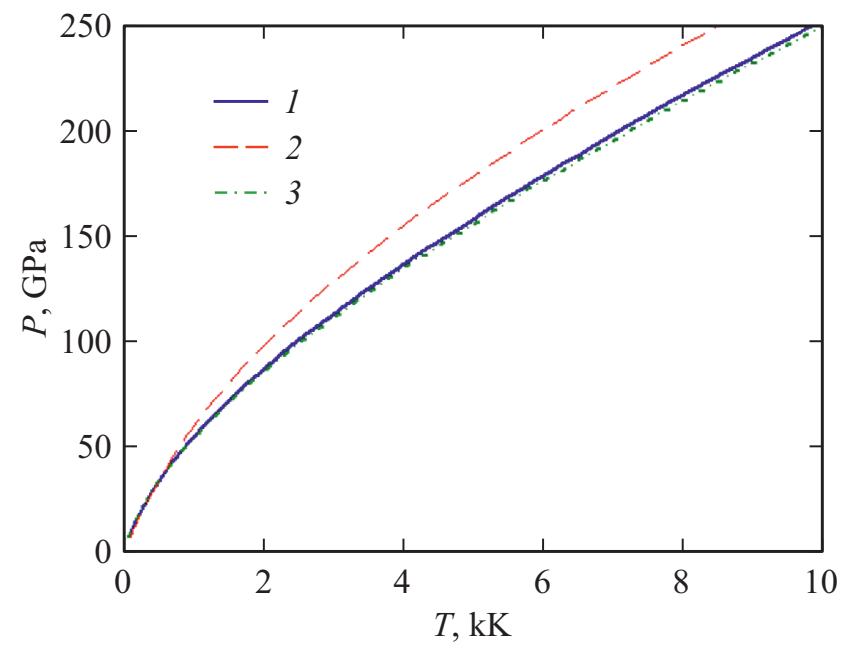

Рис. 4. Расчет значения температуры вдоль ударной адиабаты для материала и компонентов. Расчет: 1 - для смеси Elkonite $2125 \mathrm{C}$ в целом, $2-\mathrm{W}, 3-\mathrm{Cu}$. 


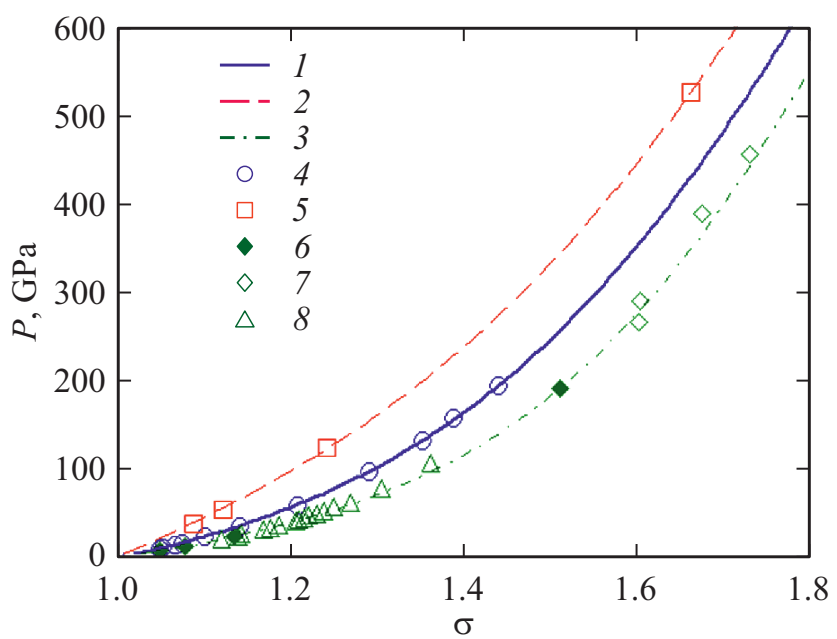

Рис. 5. Ударные адиабаты смеси Elkonite ТC10 и ее компонентов. Расчет: $1-\mathrm{TC} 10,2-\mathrm{WC}, 3-\mathrm{Cu}$. Экспериментальные данные: $4-\mathrm{TC} 10$ [23], $5-\mathrm{WC}$ [28], 6-8- Cu [24].

На рис. 3 представлены кривые, отражающие значение степени сжатия соответствующего компонента в чистом виде в сравнении со степенью сжатия, которое происходит при сжатии компонента в составе смеси. Результаты моделирования показывают, что степень сжатия чистого $\mathrm{W}$ отличается от сжатия $\mathrm{W}$ как компонента смеси при том же значении $m$. Этот результат хорошо иллюстрируется, если рассмотреть зависимости значения температуры вдоль ударной адиабаты для чистых веществ и для рассматриваемой смеси, приведенные на рис. 4. При использовании смесевого метода предполагается, что компоненты сжимаются независимо, данное предположение оправдано для сплавов и высокоплотных смесей, как это отмечено в [6]. Однако для пористых материалов при значении $\mathrm{m}$ более 1.02 , по-видимому, разница температур при нагружении чистого материала и того же материала в составе смеси приводит к появлению систематических отклонений расчетных данных от данных экспериментов [3,6]. При значении $m$ от 1 до 1.02 для сплавов результаты моделирования по рассматриваемой модели близки к расчетам по смесевой модели [6].

Проведено численное моделирование ударно-волнового нагружения, в том числе и для элконитов, содержащих в качестве компонента карбид вольфрама. Для образца из меди и карбида вольфрама (элконит ТС10) с массовыми долями $\mathrm{WC}(56) \mathrm{Cu}(44)$ [23] результаты расчетов и имеющиеся экспериментальные данные в координатах давление-сжатие показаны на рис. 5,6. Для сравнения дополнительно приведены расчеты для компонентов смеси.

Как отмечается в [2], экспериментальные данные для элконита ТС-10 согласуются с расчетными данными, которые выполнены по смесевому методу, хуже, чем для элконитов системы $\mathrm{Cu}-\mathrm{W}$. По данным, приведенным в [2], значение $\mathrm{m}$ для данного материала составля- ет 1.0026. Это означает, что данный образец можно рассматривать как сплошной, т.е. непористый. Таким образом, для композитов, включающих в свой состав компоненты с существенно разными свойствами, смесевой подход достоверно не описывает экспериментальные данные, в том числе и для сплошных образцов. Оценка значений температуры вдоль ударной адиабаты, показанная на рис. 7, демонстрирует существенную разницу между состояниями смеси и компонентов ТС-10 при воздействии на чистый материал. Для смесей такого типа учет взаимодействия компонентов необходим как для сплошных, так и для пористых образцов при расчете термодинамических параметров. Авторских подход позволяет достоверно описывать данные, полученные на основании экспериментов, и для данного материала.

Аналогичные расчеты были выполнены для металлического композита, содержащего серебро, на примере

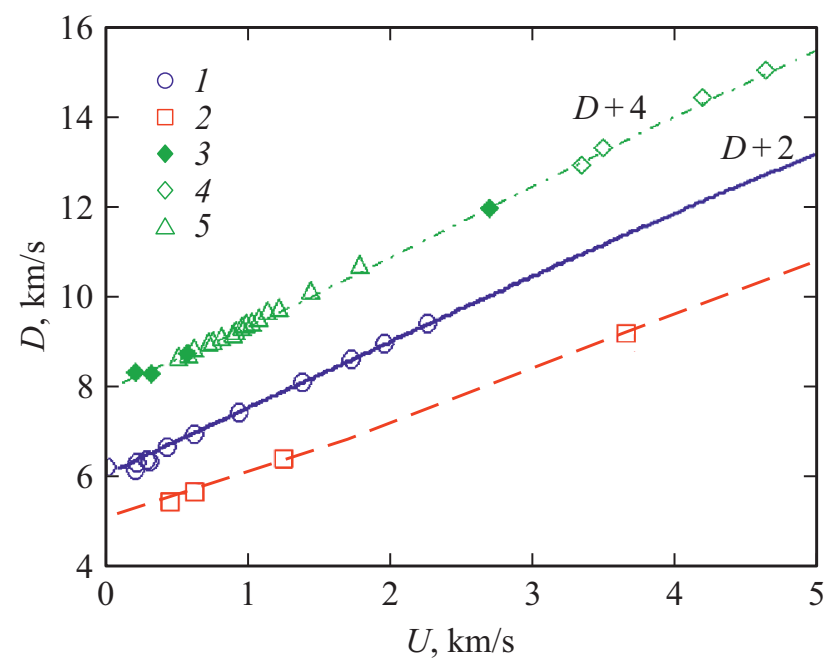

Рис. 6. Ударные адиабаты смеси Elkonite ТC10 и ее компонентов в координатах волновой скорости $D$ от массовой скорости $U: 1-$ Elkonite TC10, $2-\mathrm{WC}, 3-5-\mathrm{Cu}$.

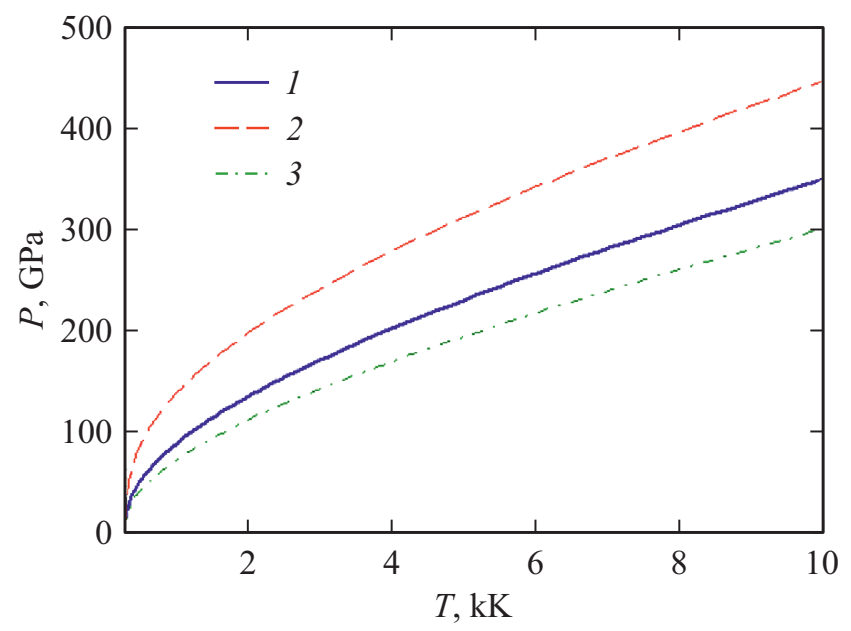

Рис. 7. Значение температуры вдоль ударной адиабаты смеси Elkonite TC10 и ее компонентов. Расчет: $1-\mathrm{WC}(56) \mathrm{Cu}(44)$, $2-\mathrm{WC}, 3-\mathrm{Cu}$. 


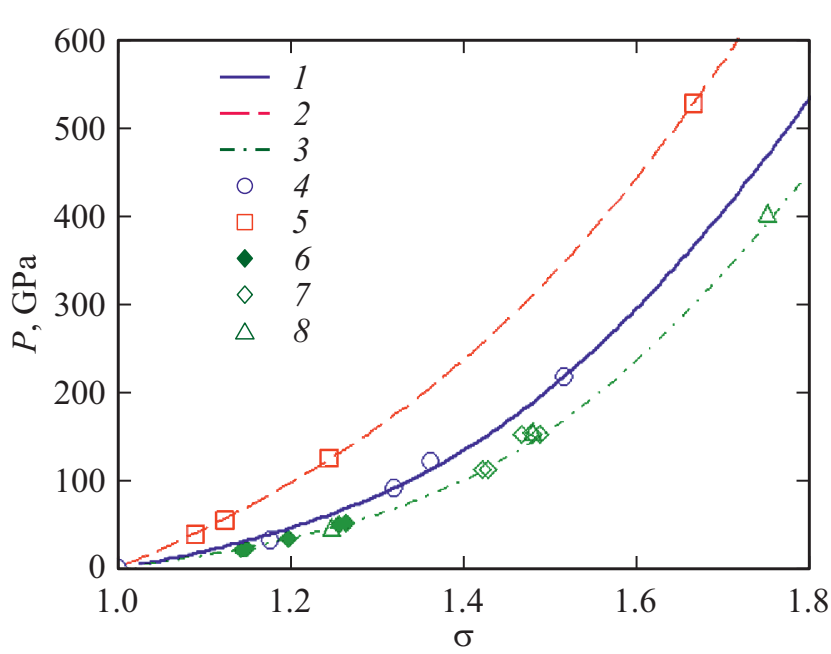

Рис. 8. Ударные адиабаты смеси Elkonite G-12 и ее компонентов в координатах $P$-сжатие $\sigma$. Расчет: $1-\mathrm{G}-12,2-\mathrm{WC}, 3-$ Ag. Экспериментальные данные: 4 - G-12 [23], 5 - WC [28], $6-\mathrm{Ag}$ [31], $7-\mathrm{Ag}$ [32], $8-\mathrm{Ag}$ [33].

элконита, содержащего WC и Ag. Исследованиям материалов такого типа посвящен ряд работ, в частности $[29,30]$. Результаты моделирования ударно-волнового нагружения для смеси серебра и карбида вольфрама (элконит G-12-WC(40) $\mathrm{Ag}(60)$ ), показаны на рис. 8. Значение пористости $m$ для G-12-1.0126, определено по данным из [23], поэтому данный материал также можно рассматривать как сплошной.

Таким образом, результаты моделирования показывают, что авторская модель позволяет описывать данные как для сплошных, так и для пористых композитов различных составов, при этом отсутствует систематическое отклонение расчетных данных от данных экспериментов, которое возникало при использовании смесевой модели. Достоверное описание имеющихся данных, полученных на основании экспериментов, позволяет использовать данную модель для подбора составов и соотношений компонентов, для получения образцов, которые позволяют достигать необходимых значений по давлению и температуре при ударно-волновых экспериментах.

Следует отметить, что, хотя в данной модели используется постоянное значение теплоемкости для компонентов, получено достоверное описание динамических параметров образцов (давление, сжатие). Как отмечено в [34], это предположение можно считать оправданным при значениях сжатия, меньших 2. Значения температуры получаются несколько завышенными при расчетах по данной модели. Для получения более достоверной информации, в том числе и о значениях температуры вдоль ударной адиабаты, разработана модификация рассматриваемого метода - модель ТЕС2 [35,36]. Для определения параметров уравнения состояния компонентов для модели ТЕС2 необходимо наличие достоверных данных по зависимости значения теплоемкости от температуры для компонентов исследуемого материала.

\section{Заключение}

Получено хорошее согласие модельных расчетов динамических параметров, таких, как давление, сжатие, волновые и массовые скорости, с данными, которые определяются на основе эксперимента в пределах его точности для металлических композитов на базе W и WC.

Рассмотренная модель позволяет учесть тот факт, что разница в температурах компонентов может быть существенной как для пористых материалов, так и для сплавов и смесей с малой пористостью. Для описания данных для сплавов и смесей различных значений пористости используются параметры, определенные для чистых материалов. Полученные результаты могут быть использованы при синтезе новых материалов для целенаправленного создания необходимых условий по значениям давления и температуры.

\section{Конфликт интересов}

Авторы заявляют об отсутствии конфликта интересов.

\section{Список литературы}

[1] М.Ю. Белякова. Изв. АН СССР Сер. Физика Земли, 1, 99 (1991).

[2] R.G. McQueen, S.P. Marsh, J.W. Taylor, J.N. Fritz, W.J. Carter. The Equation of State of Solids from Shock Wave Studies / In: High Velocity Impact Phenomena, ed. by R. Kinslow (Academic Press, NY., 1970)

[3] Б.А. Люкшин, А.В. Герасимов, Р.А. Кректулева, П.А. Люкшин. Моделирование физико-механических процессов в неоднородных конструкциях (Изд-во СО РАН, Новосибирск, 2001)

[4] A. Peikrishvili, L. Kecskes, N. Chikhradze, A. Dgebuadze. Powder Metallurgy, 46 (2), 127 (2003). DOI: $10.1179 / 003258903225005277$

[5] R.A. Krektuleva. Strength Mater., 35, 82 (2003). DOI: $10.1023 / \mathrm{A}: 1022929623159$.

[6] Р.Ф. Трунин. Исследования экстремальных состояний конденсированных веществ методом ударных волн. Уравнения Гюгонио (РФЯЦ-ВНИИЭФ, Саров, 2006)

[7] X.F. Zhang, L. Qiao, A.S. Shi, J. Zhang, Z.W. Guan. J. Appl. Phys., 110, 013506 (2011). DOI: 10.1063/1.3603018

[8] Q. Zhou, P.W. Chen, X. Gao. AIP Conf. Proc., 1426, 1347 (2012). DOI: $10.1063 / 1.3686530$

[9] Р.К. Бельхеева. ПМТФ, 53 (4), 3 (2012). [R.K. Bel'kheeva. J. Appl. Mech. Tech. Phys., 53, 471 (2012). DOI: 10.1134/S0021894412040013]

[10] B. Nayak, S.V.G. Menon. Shock Waves, 28, 141 (2018). DOI: $10.1007 / \mathrm{s} 00193-017-0717-9$

[11] B. Godibadze, A. Dgebuadze, E. Chagelishvili, G. Mamniashvili, A. Peikrishvili. J. Phys.: Conf. Ser., 987, 012027 (2018). DOI: 10.1088/1742-6596/987/1/012027

[12] А.А. Баканова, В.А. Бугаева, И.П. Дудоладов, Ю.Н. Сутулов. ПМТФ, 6, 167 (1972).

[13] Ю.Ф. Алексеев, Л.В. Альтшулер, В.П. Крупникова. ПМТФ. 4, 152 (1971). 
[14] S.L. Gavrilyuk, R. Rankine. J. Fluid Mech. 575, 495 (2007). DOI: $10.1017 / \mathrm{S} 0022112006004496$

[15] В.Ф. Куропатенко. Модели механики сплошных сред (Челяб. гос. ун-т, Челябинск, 2007)

[16] В.Ф. Куропатенко. Законы сохранения в моделях многокомпонентных сред. Сб. материалов научн. конф. „Фундаментальные и прикладные проблемы современной механики“ Томск. 2011 г. (Изд-во Том. гос. ун-та., Томск, 2012), c. 38.

[17] С.А. Кинеловский, К.К. Маевский. ТВТ, 54 (5), 716 (2016). DOI: 10.7868/S0040364416050161 [S.A. Kinelovskii, K.K. Maevskii. High Temperature, 54(5), 675 (2016). DOI: 10.1134/S0018151X16050163]

[18] С.А. Кинеловский, К.К. Маевский. ЖТФ, 86 (8), 125 (2016). [S.A. Kinelovskii, K.K. Maevskii. Tech. Phys., 61 (8), 1244 (2016). DOI: 10.1134/S1063784216080144]

[19] K.K. Maevskii. J. Phys. Conf. Series., 894, 012057 (2017). DOI: 10.1088/1742-6596/894/1/012057

[20] K.K. Maevskii. AIP Conf. Proc., 2051, 020181 (2018). DOI: $10.1063 / 1.5083424$

[21] K.K. Maevskii. Math. Montis., 45, 52 (2019). DOI: $10.20948 /$ mathmontis-2019-45-4

[22] В.В. Милявский, В.Е. Фортов, А.А Фролова, К.В Хищенко, А.А. Чарахчьян, Л.В. Шуршалов. ЖВМиМФ, 46 (5), 913 (2006). [V.V. Milyavskii, V.E. Fortov, A.A. Frolova, K.V. Khishchenko, A.A. Charakhch'yan, L.V. Shurshalov. Comput. Math. Math. Phys., 46, 873 (2006). DOI: $10.1134 / \mathrm{S} 0965542506050113$

[23] LASL Shock Hugoniot Data, ed. by S.P. Marsh (Univ. California Press, Berkeley, 1980)

[24] P.R. Levashov, K.V. Khishchenko, I.V. Lomonosov, V.E. Fortov. AIP Conf. Proc., 706, 87 (2004). http://www.ihed.ras.ru/rusbank/

[25] Р.Ф. Трунин, Л.Ф. Гударенко, М.В. Жерноклетов, Г.В. Симаков Экспериментальные данные по ударно-волновому сжатию и адиабатическому расширению конденсированных веществ (РФЯЦ-ВНИИЭФ, Саров, 2006)

[26] Физические величины, под ред. И.С.Григорьева, Е.3. Мейлихова (Энергоатомиздат, М., 1991)

[27] G.V. Belov, V.S. Iorish, V.S. Yungman. Calphad, 23 (2), 173 (1999). DOI: 10.1016/S0364- 5916(99)00023-1

[28] М.Н. Павловский. ФТТ, 12 (7), 2175 (1970).

[29] M.M. Pacheco, R.H.B. Bouma, L. Katgerman. Appl. Phys. A, 90, 159 (2008). DOI: 10.1007/s00339-007-4245-9

[30] N. Ray, B. Kempf, T. Mützel, L. Froyen, K. Vanmeensel, J. Vleugels. Mater. Des., 85, 412 (2015). DOI: $10.1016 /$ j.matdes.2015.07.006

[31] J.M. Walsh, M.H. Rice, R.G. Mcqueen, F.L. Yarger. Phys. Rev., 108, 196 (1957).

[32] R.G. McQueen, S.P. Marsh. J. Appl. Phys., 31, 1253 (1960).

[33] Л.В. Альтшулер, К.К. Крупников, М.И. Бражник. ЖЭТФ, $34(4), 886$ (1958).

[34] А.В. Острик. Конструкции из композиционных материалов, 2, 48 (2018).

[35] К.К. Маевский, С.А. Кинеловский. ЖТФ, 89(8), 1158 (2019). [K.K. Maevskii, S.A. Kinelovskii. Tech. Phys., 64, 1090 (2019).] DOI: 10.1134/S1063784219080127

[36] K.K. Maevskii. J. Phys.: Conf. Ser. 1385, 012001 (2019). DOI: $10.1088 / 1742-6596 / 1385 / 1 / 012001$ 